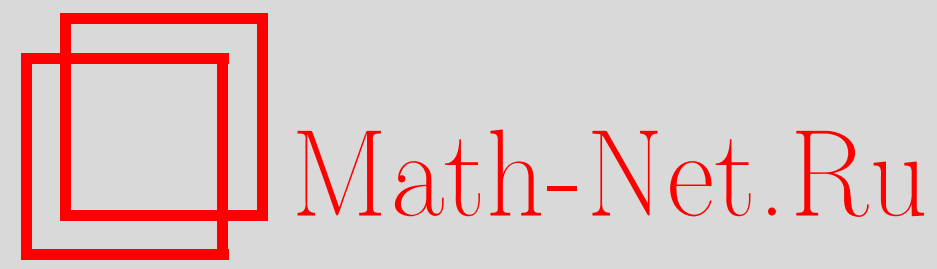

C. Berzin-Joseph, J. R. León, Weak convergence of the integrated number of level crossings to the local time for Wiener processes, Теория вероятн. и ее примен., 1997, том 42, выпуск 4, 757-771

DOI: https://doi.org/10.4213/tvp2289

Использование Общероссийского математического портала Math-Net.Ru подразумевает, что вы прочитали и согласны с пользовательским соглашением http://www . mathnet.ru/rus/agreement

Параметры загрузки:

IP : 54.210 .77 .194

26 апреля 2023 г., 15:59:10

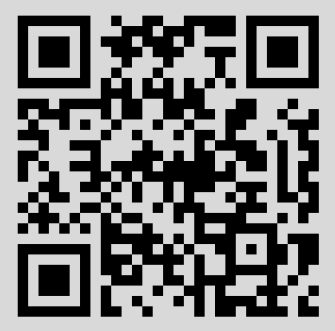


(C) 1997 г. $\quad$ BERZIN-JOSEPH C. ${ }^{*}$, LEÓN J. R.**

\section{WEAK CONVERGENCE OF THE INTEGRATED NUMBER OF LEVEL CROSSINGS TO THE LOCAL TIME FOR WIENER PROCESSES}

Пусть $\left\{X_{t}, t \in[0,1]\right\}$ есть стандартный винеровский процесс, определенный на $(\Omega, \mathcal{A}, \mathbf{P})$. Рассмотрим упорядочивающий процесс $X_{t}^{\varepsilon}=\varphi_{\varepsilon} * X_{t}$, где $\varphi_{\varepsilon}(t)=(1 / \varepsilon) \varphi(t / \varepsilon)$ есть ядро, сходящееся к дельтафункции Дирака при $\varepsilon \rightarrow 0$. В статье изучается сходимость

$$
Z_{\varepsilon}(f)=\varepsilon^{-1 / 2} \int_{-\infty}^{+\infty}\left[\frac{N^{X^{c}}(x)}{c(\varepsilon)}-L_{X}(x)\right] f(x) d x
$$

когда $\varepsilon$ стремится к нулю, здесь $N^{X^{\varepsilon}}(x)$ есть число пересечений процессом $X^{\varepsilon}$ уровня $x$ в промежутке $[0,1]$, а $L_{X}(x)$ есть локальное время пребывания $X$ в $x$ на отрезке $[0,1]$. Как следствие предложенного метода, получен результат о слабой сходимости для приращений процесса $X$.

Ключевые слова и фразы: винеровский процесс, локальное время, пересечения уровня, приращения.

1. Introduction. Let $X_{t}=\{X(t, \omega), t \in[0,1], \omega \in \Omega\}$ be a standard Wiener process. For each $t$ and $\varepsilon>0$ define $\Delta_{\varepsilon}(t)=\varepsilon^{-1 / 2}\left(X_{t+\varepsilon}-X_{t}\right)$, the normalized increments of the process. If we fix a trajectory and consider $\Delta_{\varepsilon}(t)$ as a random variable (r.v.) on $t$ with Lebesgue's measure $\lambda$ then, as $M$. Wschebor showed [10], for almost every trajectory, this variable converges in distribution, as $\varepsilon$ goes to zero, to a Gaussian distribution:

$$
F_{\varepsilon}(t, x)=\lambda\left\{s \leqslant t: \Delta_{\varepsilon}(s) \leqslant x\right\} \longrightarrow t \Phi(x) \text { as } \varepsilon \rightarrow 0
$$

for almost every (a.e.) $\omega \in \Omega$, where $\Phi$ is the standard Gaussian distribution function.

It is interesting now to find out at what speed this convergence takes place. Moreover, one would like to know if

$$
K_{\varepsilon}(t, x)=F_{\varepsilon}(t, x)-t \Phi(x)
$$

*Université Versailles-Saint-Quentin en Yvelines; Université Paris-Sud, Département de Mathématiques, Laboratoire de Statistiques Orsay, Bât 425, Centre d'Orsay, 91405 Orsay Cédex, France.

${ }^{* *}$ U. C. V. Facultad de Ciencias, Departamento de Matemáticas; A. P. 47197, Los Chaguaramos, Caracas 1041-A, Venezuela. 
conveniently normalized, converges weakly. To this end take $\varphi(t)=I_{[-1,0]}(t)$ and define $X_{t}^{\varepsilon}=\varphi_{\varepsilon} * X_{t}$, the convolution of the process with $\varphi_{\varepsilon}(t)=$ $(1 / \varepsilon) \varphi(t / \varepsilon)$. Then (cf. [10]) $\Delta_{\varepsilon}(t)=\varepsilon^{1 / 2} \dot{X}_{t}^{\varepsilon}$. Thus, we will work in a more general setting by considering the process $\varepsilon^{1 / 2} \dot{X}_{t}^{\varepsilon}$ for different functions $\varphi$ instead of $\Delta_{\varepsilon}(t)$.

A related, and more involved problem, will also be considered. Let $N^{X^{\varepsilon}}(x)$ be the number of crossings of the level $x$ by the process $X^{\varepsilon}$ in the interval $[0,1]$, and $L_{X}(x)$ be the local time of $X$ at level $x$. Define the r.v.

$$
\int_{-\infty}^{\infty} f(x) N^{X^{\varepsilon}}(x)\left(\frac{\pi}{2} \varepsilon\right)^{1 / 2}\|\varphi\|_{2}^{-1} d x
$$

whose almost sure (a.s:) limit is $\int_{-\infty}^{\infty} f(x) L_{X}(x) d x$ (cf. [10]) where $f$ satisfies certain regularity conditions. We study the convergence in distribution of the normalized difference between these two variables, which we call $Z_{\varepsilon}(f)$. We show in Theorem 1 that $Z_{\varepsilon}(f)$ converges in distribution, when $\varepsilon$ tends to zero to $\sigma \int_{0}^{1} f\left(X_{s}\right) d W_{s}$ where $W$ is a Brownian motion independent of $X$ and $\sigma$ is a positive constant.

As a consequence of the proof of the Theorem 1 we prove that

$$
B_{\varepsilon}(t, x)=\varepsilon^{-1 / 2} K_{\varepsilon}(t, x) \longrightarrow \eta(t, x)
$$

in distribution in $C[0,1]$ for each $x$ when $\varepsilon$ tends to zero, where $\eta(t, x)$ is a Gaussian process with independent increments in $t$ and a variance that depends on $x$. This answers the two questions raised above.

The authors in another article [1] considered the same problem for stationary Gaussian processes whose covariance behaves like $r(t)=1-L(t)|t|^{2 \alpha}$ with $0<\alpha<1$. The Ornstein-Uhlenbeck process is a particular case $\left(\alpha=\frac{1}{2}\right)$ and the results are similar to those of the Wiener process.

There exists a vast literature on results involving second order approximations for Brownian local times. It is worth citing, among the first contributions, the work of Kasahara [7], where the author obtains a weak limit for the normalized difference between the number of times that the reflected Brownian motion crosses down from $\varepsilon$ to 0 and the local time in zero. Similar results were also studied by Borodin in two papers [4], [5], and in several works by Csaki, Csörgö, Földes and Révész, wonderfully summarized in a recent paper [3]. For more information one can read the survey article [6] and the references cited therein.

In these papers there also appears a Brownian motion $W$ which is independent of the original one. In the modern literature, such a process is the result of applying Knight's famous theorem (cf. [8, p. 172]) on the independence of two continuous martingales whose mixed bracket equals zero. We will not be able to use this type of method in our work given the anticipating character of the process $X_{t}^{\varepsilon}$. Instead, we will adopt an ad hoc method, whose description we will give in Section 4 below. 
We remark, however, that in our work we consider a regularization by convolution of the Brownian motion, and, to our knowledge, the weak limit, after normalization, of the difference

$$
\left(N^{X^{c}}(x)\left(\frac{\pi}{2} \varepsilon\right)^{1 / 2}\|\varphi\|_{2}^{-1}-L_{X}(x)\right)
$$

is still an open problem.

\section{Assumptions and notation.}

(H1) On the process $X:\left\{X_{t}, t \in[0,1]\right\}$ is a Wiener process. In what follows, we shall suppose that $X(t)$ is defined for all $t \in \mathbf{R}$, setting $X(t)=0$ when $t \notin \mathbf{R}^{+}$.

(H2) On the kernel $\varphi: \operatorname{supp} \varphi \subseteq[-1,1], \varphi$ is the distribution function of a (signed) measure $d \varphi(y)$ which has bounded total variation, $\int_{-1}^{+1} \varphi(t) d t=1$. We define $\psi(u)=\widetilde{\varphi} * \tilde{\varphi}(-u)$ where $\tilde{\varphi}(u)=\varphi(-u), u \in \mathbf{R}$.

(H3) O n the function $f: f \in C^{2}$ and $f^{\prime \prime}$ is bounded.

We define

$$
g(x)=\sqrt{\frac{\pi}{2}}|x|-1=\sum_{n=1}^{\infty} a_{2 n} H_{2 n}(x),
$$

where $\left\{H_{n}, n \geqslant 0\right\}$ are Hermite's polynomials, orthogonal with respect to the standard Gaussian distribution and with leading coefficient equal to 1. $\phi$ denotes the standard Gaussian density.

We have

$$
\dot{X}_{t}^{\varepsilon}=\frac{1}{\varepsilon} \int_{-\infty}^{t / \varepsilon} X_{t-\varepsilon y} d \varphi(y),
$$

and we define $\dot{Y}_{t}^{\varepsilon}=\varepsilon^{1 / 2} \dot{X}_{t}^{\varepsilon}\|\varphi\|_{2}^{-1}$,

$$
Z_{\varepsilon}(f)=\varepsilon^{-1 / 2} \int_{-\infty}^{\infty} f(x)\left[\frac{N^{X^{\varepsilon}}(x)}{c(\varepsilon)}-L_{X}(x)\right] d x
$$

with $c(\varepsilon)=\sqrt{2 /(\pi \varepsilon)}\|\varphi\|_{2}, \theta(x)=\psi(x)\|\varphi\|_{2}^{-2}, x \in \mathbf{R}, \sigma_{0}^{2}=\int_{0}^{1} \mathrm{E}\left[f^{2}\left(X_{s}\right)\right] d s$. Ent $\{z\}, z \in \mathbf{R}$, is the integer part of $z$.

$\mathrm{R} \mathrm{e} \mathrm{m}$ a r k. The process $\dot{Y}_{t}^{\varepsilon}$ has unit variance on $\{t>\varepsilon\}$.

Throughout the paper, $C$ shall stand for a generic constant, whose value may change during a proof.

\section{Results.}

Theorem 1. Under assumptions $(\mathrm{H} 1),(\mathrm{H} 2)$ and $(\mathrm{H} 3)$ the process $Z_{\varepsilon}(f)$ converges weakly when $\varepsilon$ tends to zero towards a r.v. $Y \in L^{2}(\Omega)$ and the conditional distribution $\left(Y \mid X_{s}, 0 \leqslant s \leqslant 1\right)$ is Gaussian with zero mean and 
random variance equal to

$$
\sigma^{2} \int_{0}^{1} f^{2}\left(X_{s}\right) d s \quad \text { where } \sigma^{2}=\int_{-\infty}^{+\infty} \mathbf{E}\left[g\left(Z_{1}(u)\right) g\left(Z_{2}(u)\right)\right] d u
$$

and $\left(Z_{1}(u), Z_{2}(u)\right)$ is a Gaussian vector with zero mean and covariance matrix

$$
\Gamma(u)=\left(\begin{array}{cc}
1 & \theta(u) \\
\theta(u) & 1
\end{array}\right)
$$

$\mathrm{R}$ e m a r k s. a) Given the $\sigma$-algebra generated by $\left\{X_{s}, 0 \leqslant s \leqslant 1\right\}$, the limit variable $Y$ is the stochastic integral of $f\left(X_{s}\right)$ with respect to a Brownian motion $\sigma W$. This last process is the limit of

$$
S_{t}^{\varepsilon}=\frac{1}{\sqrt{\varepsilon}} \int_{0}^{t} g\left(\dot{Y}_{s}^{\varepsilon}\right) I_{[M \varepsilon, 1]}(s) d s
$$

where $M>0$ is «large enough» (see the proofs 4.c. $\alpha$. and 4.c. $\beta$.), i.e., $\sigma \int_{0}^{1} f\left(X_{s}\right) d W(s)$.

b) Another expression for $\sigma^{2}$ is

$$
\sigma^{2}=\int_{-2}^{2} \sum_{n=1}^{\infty} a_{2 n}^{2}(2 n) ! \theta^{2 n}(u) d u
$$

The next result concerns the increments. Let $\xi$ be a standard Gaussian r.v. and define

$$
\zeta_{\varepsilon}(t)=\int_{0}^{t}\left(\left|\Delta_{\varepsilon}(u)\right|^{\beta}-\mathbf{E}|\xi|^{\beta}\right) d u
$$

Theorem 2. Under hypothesis (H1); (H2) and (H3) we have

$$
\varepsilon^{-1 / 2} \zeta_{\varepsilon}(\bullet) \longrightarrow \sigma_{\beta} W_{\bullet}
$$

in distribution in $C[0,1]$ and

$$
B_{\varepsilon}(\bullet, x) \longrightarrow \eta(\bullet, x)
$$

$\eta(t, x)$ is a Gaussian process with independent increments in $t$ and a variance linear on $t$ that depends on $x$. The convergence here is in distribution in $C[0,1]$ for each $x \in \mathbf{R}$.

\section{Proofs.}

Proof of $\mathrm{Th}$ e ore $\mathrm{m} 1$. Using as in [1] the formula

$$
\int_{-\infty}^{\infty} f(x) N^{X^{\varepsilon}}(x) d x=\int_{0}^{1} f\left(X_{s}^{\varepsilon}\right)\left|\dot{X}_{s}^{\varepsilon}\right| d s
$$


we have the decomposition of $Z_{\varepsilon}(f)$ :

$$
Z_{\varepsilon}(f)=\varepsilon^{-1 / 2} \int_{0}^{1} f\left(X_{s}^{\varepsilon}\right) g\left(\dot{Y}_{s}^{\varepsilon}\right) d s+\varepsilon^{-1 / 2} \int_{0}^{1}\left[f\left(X_{s}^{\varepsilon}\right)-f\left(X_{s}\right)\right] d s .
$$

Splitting the integrals in $[0, M \varepsilon]$ and $[M \varepsilon, 1]$, where $M>0$ will be chosen later, we get

$$
\begin{aligned}
Z_{\varepsilon}(f)= & \varepsilon^{-1 / 2} \int_{0}^{M \varepsilon} f\left(X_{s}^{\varepsilon}\right) g\left(\dot{Y}_{s}^{\varepsilon}\right) d s+\varepsilon^{-1 / 2} \int_{M \varepsilon}^{1} f\left(X_{s}^{\varepsilon}\right) g\left(\dot{Y}_{s}^{\varepsilon}\right) d s \\
& +\varepsilon^{-1 / 2} \int_{0}^{M \varepsilon}\left[f\left(X_{s}^{\varepsilon}\right)-f\left(X_{s}\right)\right] d s+\varepsilon^{-1 / 2} \int_{M \varepsilon}^{1}\left[f\left(X_{s}^{\varepsilon}\right)-f\left(X_{s}\right)\right] d s \\
= & I_{1}+T_{1}+I_{2}+T_{2} .
\end{aligned}
$$

The proof will proceed as follows: we prove in a. that $I_{1}, I_{2}$ and $T_{2}$ converge to zero in $L^{2}(\Omega)$ when $\varepsilon$ goes to zero; hence the important term in the development of $Z_{\varepsilon}(f)$ is $T_{1}$ and we show in b. that $\mathbf{E}\left[T_{1}^{2}\right]$ converges to $\sigma^{2} \sigma_{0}^{2}$. In c. we prove that $\left(X_{t}^{\varepsilon}, S_{t}^{\varepsilon}\right)$ converges weakly to $\left(X_{t}, \sigma W_{t}\right)$. Furthermore the processes $X$ and $W$ are independent. In d. we will consider the convergence of $T_{1}$. We study first the convergence of the following discrete version of $T_{1}$ :

$$
Z_{\varepsilon}^{n}(f)=\varepsilon^{-1 / 2} \sum_{i=1}^{n} f\left(X_{(i-1) / n}^{\varepsilon}\right) \int_{(i-1) / n}^{i / n} g\left(\dot{Y}_{s}^{\varepsilon}\right) I_{[M \varepsilon, 1]}(s) d s .
$$

Define

$$
Z^{n}(f)=\sum_{i=1}^{n} f\left(X_{(i-1) / n}\right)\left[W_{i / n}-W_{(i-1) / n}\right] \sigma,
$$

we know from c. that $Z_{\varepsilon}^{n}(f) \rightarrow Z^{n}(f)$, weakly as $\varepsilon$ goes to zero. On the other hand there exists a r.v. $Y \in L^{2}(\Omega)$ such that: $Z^{n}(f) \rightarrow Y$ in $L^{2}(\Omega)$ when $n$ goes to infinity; furthermore, we can characterize this variable using the asymptotic independence between $X$ and $W$ :

$$
\mathcal{L}\left(Y \mid X_{s}, 0 \leqslant s \leqslant 1\right)=\mathcal{N}\left(0, \sigma^{2} \int_{0}^{1} f^{2}\left(X_{s}\right) d s\right) .
$$

Hence, to prove the convergence of $T_{1}$, it will be enough to prove that

$$
\lim _{n \rightarrow \infty} \lim _{\varepsilon \rightarrow 0}\left\|T_{1}-Z_{\varepsilon}^{n}(f)\right\|_{2}=0 .
$$

a. Preli min a r y res u l t s. Let $z$ be a continuous function verifying $|z(x)| \leqslant C\left(1+|x|^{n}\right)$ for some $n \in \mathbf{N}$. The function $g$ defined in Section 2 verifies this condition.

Lemma 1. For all $0 \leqslant s \leqslant 1, p \in \mathbf{N}$ and $\varepsilon>0$,

$$
\sup \left\{\mathbf{E}\left[f^{2 p}\left(X_{s}^{\varepsilon}\right)\right], \mathbf{E}\left[z^{2 p}\left(\dot{Y}_{s}^{\varepsilon}\right)\right], \mathbf{E}\left[f^{2 p}\left(X_{s}\right)\right], \mathbf{E}\left[f^{2 p}\left(X_{s}\right)\right]\right\} \leqslant C .
$$


P r o o f. Using the Taylor development of $f$ we obtain

$$
f\left(X_{s}^{\varepsilon}\right)=f(0)+X_{s}^{\varepsilon} f^{\prime}(0)+\frac{1}{2}\left(X_{s}^{\varepsilon}\right)^{2} f^{\prime \prime}(\alpha) .
$$

By using that $f^{\prime \prime}$ is bounded we get

$$
\mathbf{E}\left[f^{2 p}\left(X_{s}^{\varepsilon}\right)\right] \leqslant C \mathbf{E}\left[P\left(X_{s}^{\varepsilon}\right)\right]
$$

where $P$ is a polynomial. But

$$
\mathbf{E}\left[X_{s}^{\varepsilon}\right]^{2}=2 \int_{-\infty}^{s / \varepsilon} \int_{u}^{s / \varepsilon} \varphi(w)[s-\varepsilon w] d w \varphi(u) d u \leqslant C[s+1] \leqslant C
$$

because $s \leqslant 1$. Similarly, $\mathbf{E}\left[f^{2 p}\left(X_{s}\right)\right]$ and $\mathbf{E}\left[f^{2 p}\left(X_{s}\right)\right]$ are bounded.

Recall that $\dot{Y}_{s}^{\varepsilon}=\sqrt{\varepsilon} \dot{X}_{s}^{\varepsilon}\|\varphi\|_{2}^{-1}$; using that $\mathbf{E}\left[\sqrt{\varepsilon} \dot{X}_{s}^{\varepsilon}\right]^{2}=\int_{-\infty}^{s / \varepsilon} \varphi^{2}(u) d u \leqslant$ $\|\varphi\|_{2}^{2}$ and $z^{2 p}(x) \leqslant C\left[|x|^{2 p}+1\right]$, for all $x \in \mathbf{R}, \mathbf{E}\left(z^{2 p}\left(\dot{Y}_{s}^{\varepsilon}\right)\right)$ is bounded and Lemma 1 holds.

Consider

$$
I_{1}=\varepsilon^{-1 / 2} \int_{0}^{M \varepsilon} f\left(X_{s}^{\varepsilon}\right) g\left(\dot{Y}_{s}^{\varepsilon}\right) d s ; \quad \mathbf{E}\left[I_{1}^{2}\right] \leqslant M \int_{0}^{M \varepsilon} \mathbf{E}\left[f^{2}\left(X_{s}^{\varepsilon}\right) g^{2}\left(\dot{Y}_{s}^{\varepsilon}\right)\right] d s
$$

Using Schwarz's inequality and Lemma 1 , we have $\mathbf{E}\left[I_{1}^{2}\right] \leqslant C \varepsilon$ where $C$ depends on $M$.

Similarly, $\mathbf{E}\left[I_{2}^{2}\right] \leqslant C \varepsilon$.

For $T_{2}=\varepsilon^{-1 / 2} \int_{M \varepsilon}^{1}\left[f\left(X_{s}^{\varepsilon}\right)-f\left(X_{s}\right)\right] d s$, using Taylor's development and calling $Q_{s}^{\varepsilon}=\varepsilon^{-1 / 2}\left[X_{s}^{\varepsilon}-X_{s}\right]$, we have

$$
\varepsilon^{-1 / 2}\left[f\left(X_{s}^{\varepsilon}\right)-f\left(X_{s}\right)\right]=Q_{s}^{\varepsilon} f^{\prime}\left(X_{s}\right)+\frac{1}{2} \sqrt{\varepsilon}\left(Q_{s}^{\varepsilon}\right)^{2} f^{\prime \prime}\left(\alpha_{1}\right) .
$$

The asymptotic law of $Q_{s}^{\varepsilon}$ is $\mathcal{N}\left(0, C_{2}\right)$ where

$$
C_{2}=2 \int_{-\infty}^{+\infty}\left[\int_{0}^{u} w \varphi(w) d w\right] \varphi(u) d u
$$

To prove this it is enough to calculate covariances, but if $M \geqslant 1$ we have $s \geqslant \varepsilon$, then

$$
\mathbf{E}\left[X_{s}^{\varepsilon}\right]^{2}=s-\varepsilon C_{1} \quad \text { with } C_{1}=2 \int_{-\infty}^{+\infty}\left[\int_{u}^{+\infty} w \varphi(w) d w\right] \varphi(u) d u
$$

and

$$
\mathbf{E}\left[X_{s}^{\varepsilon} X_{s}\right]=s-\varepsilon C_{3} \quad \text { with } C_{3}=\int_{0}^{+\infty} w \varphi(w) d w
$$


see [9] for the calculus of variances and covariances. Therefore,

$$
\begin{aligned}
\mathbf{E}\left[T_{2}^{2}\right] \leqslant & C\left[\int_{M \varepsilon}^{1} \int_{M \varepsilon}^{1} \mathbf{E}\left[Q_{s}^{\varepsilon} Q_{t}^{\varepsilon} f^{\prime}\left(X_{s}\right) f^{\prime}\left(X_{t}\right)\right] d s d t\right. \\
& \left.+\varepsilon \mathbf{E}\left[\int_{M \varepsilon}^{1}\left(Q_{s}^{\varepsilon}\right)^{2} f^{\prime \prime}\left(\alpha_{1}\right) d s\right]^{2}\right] .
\end{aligned}
$$

For the first term, by computing covariances, one can show that

$$
\left(Q_{s}^{\varepsilon}, Q_{t}^{\varepsilon}, X_{s}, X_{t}\right) \longrightarrow\left(Y_{1}, Y_{2}, X_{s}, X_{t}\right)
$$

in distribution, where $Y_{1}$ and $Y_{2}$ are independent $\mathcal{N}\left(0, C_{2}\right)$. The vectors $\left(Y_{1}, Y_{2}\right)$ and $\left(X_{s}, X_{t}\right)$ are also independent.

By using uniform integrability (bounding $\mathbf{E}\left[Q_{s}^{\varepsilon} Q_{t}^{\varepsilon} f^{\prime}\left(X_{s}\right) f^{\prime}\left(X_{t}\right)\right]^{2}$ uniformly in $\varepsilon$ and using Lemma 1) we prove that $\mathbf{E}\left[Q_{s}^{\varepsilon} Q_{t}^{\varepsilon} f^{\prime}\left(X_{s}\right) f^{\prime}\left(X_{t}\right)\right] \longrightarrow 0$ when $\varepsilon$ goes to zero. Hence the Dominated Convergence Theorem gives the convergence to zero. For the second term using that $f^{\prime \prime}$ is bounded we get $O(\varepsilon)$.

b. As y mptotic second order moment of $T_{1}$.

We have

$$
\begin{aligned}
T_{1} & =\varepsilon^{-1 / 2} \int_{M \varepsilon}^{1} f\left(X_{s}^{\varepsilon}\right) g\left(\dot{Y}_{s}^{\varepsilon}\right) d s \\
\mathbf{E}\left[T_{1}^{2}\right] & =\varepsilon^{-1} \int_{M \varepsilon}^{1} \int_{M \varepsilon}^{1} \mathbf{E}\left[f\left(X_{s}^{\varepsilon}\right) f\left(X_{t}^{\varepsilon}\right) g\left(\dot{Y}_{s}^{\varepsilon}\right) g\left(\dot{Y}_{t}^{\varepsilon}\right)\right] d t d s .
\end{aligned}
$$

We consider the change of variable: $t=s+\varepsilon u$ :

$$
\mathrm{E}\left[T_{1}^{2}\right]=\int_{M \varepsilon}^{1} \int_{(M \varepsilon-s) / \varepsilon}^{(1-s) / \varepsilon} \mathbf{E}\left[f\left(X_{s}^{\varepsilon}\right) f\left(X_{s+\varepsilon u}^{\varepsilon}\right) g\left(\dot{Y}_{s}^{\varepsilon}\right) g\left(\dot{Y}_{s+\varepsilon u}^{\varepsilon}\right)\right] d u d s .
$$

There are three possible cases:

(1) $(M+2) \varepsilon \leqslant s \leqslant 1-2 \varepsilon$

(2) $1-2 \varepsilon \leqslant s$,

(3) $s \leqslant(M+2) \varepsilon$.

$\mathrm{C}$ a s e 1 . The integral can be written as $J_{1}+J_{2}+J_{3}$, where

$$
\begin{aligned}
& J_{1}=\int_{(M+2) \varepsilon}^{1-2 \varepsilon} \int_{-2}^{2} \mathbf{E}\left[f\left(X_{s}^{\varepsilon}\right) f\left(X_{s+\varepsilon u}^{\varepsilon}\right) g\left(\dot{Y}_{s}^{\varepsilon}\right) g\left(\dot{Y}_{s+\varepsilon u}^{\varepsilon}\right)\right] d u d s, \\
& J_{2}=\int_{(M+2) \varepsilon}^{1-2 \varepsilon} \int_{2}^{(1-s) / \varepsilon} \mathbf{E}\left[f\left(X_{s}^{\varepsilon}\right) f\left(X_{s+\varepsilon u}^{\varepsilon}\right) g\left(\dot{Y}_{s}^{\varepsilon}\right) g\left(\dot{Y}_{s+\varepsilon u}^{\varepsilon}\right)\right] d u d s, \\
& J_{3}=\int_{(M+2) \varepsilon}^{1-2 \varepsilon} \int_{(M \varepsilon-s) / \varepsilon}^{-2} \mathbf{E}\left[f\left(X_{s}^{\varepsilon}\right) f\left(X_{s+\varepsilon u}^{\varepsilon}\right) g\left(\dot{Y}_{s}^{\varepsilon}\right) g\left(\dot{Y}_{s+\varepsilon u}^{\varepsilon}\right)\right] d u d s .
\end{aligned}
$$


F o r $J_{1}$. Using Lemma 1 we prove, by the Dominated Convergence Theorem and uniform integrability, that $J_{1}$ converges to $\sigma^{2} \sigma_{0}^{2}$ when $\varepsilon$ goes to zero.

F o r $J_{2}$. Let $\Sigma_{\varepsilon}(s, u)$ be the covariance matrix of the Gaussian vector $\left(X_{s}^{\varepsilon}, X_{s+\varepsilon u}^{\varepsilon}, \dot{Y}_{s}^{\varepsilon}, \dot{Y}_{s+\varepsilon u}^{\varepsilon}\right)$. Since $u \geqslant 2$, it is easy to check that

$\Sigma_{\varepsilon}(s, u)=\left(\begin{array}{cccc}s-\varepsilon C_{1} & s-\varepsilon D & 2^{-1} \sqrt{\varepsilon}\|\varphi\|_{2}^{-1} & 0 \\ s-\varepsilon D & s+\varepsilon u-\varepsilon C_{1} & \sqrt{\varepsilon}\|\varphi\|_{2}^{-1} & 2^{-1} \sqrt{\varepsilon}\|\varphi\|_{2}^{-1} \\ 2^{-1} \sqrt{\varepsilon}\|\varphi\|_{2}^{-1} & \sqrt{\varepsilon}\|\varphi\|_{2}^{-1} & 1 & 0 \\ 0 & 2^{-1} \sqrt{\varepsilon}\|\varphi\|_{2}^{-1} & 0 & 1\end{array}\right)$

where

$$
D=\int_{-\infty}^{+\infty} u \varphi(u) d u
$$

We now fix $s$ and $u$ and consider the change of variables

$$
\begin{aligned}
X_{s}^{\varepsilon} & =Z_{1} ; \quad \dot{Y}_{s}^{\varepsilon}=Z_{2} ; \quad \dot{Y}_{s+\varepsilon u}^{\varepsilon}=Z_{3} \\
X_{s+\varepsilon u}^{\varepsilon} & =\beta_{1} Z_{1}+\beta_{2} Z_{2}+\beta_{3} Z_{3}+\beta_{4} Z_{4}
\end{aligned}
$$

with $Z_{3}$ independent of $\left(Z_{1}, Z_{2}\right)$ and $Z_{4}$ independent of $\left(Z_{1}, Z_{2}, Z_{3}\right)$, where

$$
\begin{aligned}
& \beta_{1}=\left[s-\varepsilon D-2^{-1} \varepsilon\|\varphi\|_{2}^{-2}\right]\left[s-\varepsilon C_{1}-4^{-1} \varepsilon\|\varphi\|_{2}^{-2}\right]^{-1} \\
& \beta_{2}=\left(2^{-1} s-\varepsilon C_{1}\right) \varepsilon^{1 / 2}\|\varphi\|_{2}^{-1}\left[s-\varepsilon C_{1}-4^{-1} \varepsilon\|\varphi\|_{2}^{-2}\right]^{-1} \\
& \beta_{3}=2^{-1} \varepsilon^{1 / 2}\|\varphi\|_{2}^{-1}
\end{aligned}
$$

and

$$
\beta_{4}=\left[s+\varepsilon u-\varepsilon C_{1}-\beta_{1}^{2}\left(s-\varepsilon C_{1}\right)-\beta_{2}^{2}-\beta_{1} \beta_{2} \varepsilon^{1 / 2}\|\varphi\|_{2}^{-1}-\beta_{3}^{2}\right]^{1 / 2} .
$$

Since $\beta_{2}$ and $\beta_{3}$ converge to zero when $\varepsilon$ goes to zero, we develop $f$ in a neighbourhood of $\beta_{1} z_{1}+\beta_{4} z_{4}$; using that

$$
\int_{-\infty}^{+\infty} g\left(z_{3}\right) \phi\left(z_{3}\right) d z_{3}=\int_{-\infty}^{+\infty} z_{3} g\left(z_{3}\right) \phi\left(z_{3}\right) d z_{3}=0
$$

and calling $\phi\left(z_{1}, z_{2}, s, \varepsilon\right)$ the joint Gaussian density of variables $\left(X_{s}^{\varepsilon}, \dot{Y}_{s}^{\varepsilon}\right)$, one has

$$
J_{2}=\int_{(M+2) \varepsilon}^{1-2 \varepsilon}\left[\int_{\mathbf{R}^{2}} G_{\varepsilon}\left(z_{1}, z_{2}, s\right) F_{\varepsilon}\left(z_{1}, z_{2}, s\right) d z_{1} d z_{2}\right] d s
$$

where

$$
G_{\varepsilon}\left(z_{1}, z_{2}, s\right)=f\left(z_{1}\right) g\left(z_{2}\right) \phi\left(z_{1}, z_{2}, s, \varepsilon\right) I_{[(M+2) \varepsilon, 1-2 \varepsilon]}(s)
$$


and

$$
\begin{aligned}
F_{\varepsilon}\left(z_{1}, z_{2}, s\right)=\int_{2}^{(1-s) / \varepsilon}[ & \int_{\mathbf{R}^{2}} g\left(z_{3}\right) \frac{f^{\prime \prime}\left(\theta_{u}\right)}{2}\left(\beta_{2} z_{2}+\beta_{3} z_{3}\right)^{2} \\
& \left.\times \phi\left(z_{3}\right) \phi\left(z_{4}\right) d z_{3} d z_{4}\right] d u I_{[(M+2) \varepsilon, 1-2 \varepsilon]}(s) .
\end{aligned}
$$

Using that $f^{\prime \prime}$ is bounded, that $\beta_{2}$ and $\beta_{3}$ are smaller than $C \sqrt{\varepsilon}$ (since $s \geqslant M \varepsilon, s-\varepsilon C_{1}-4^{-1} \varepsilon\|\varphi\|_{2}^{-1} \geqslant 2^{-1} s$ for $M$ large enough), that $g$ has finite moments of all orders with respect to the measure $\phi(x) d x$, Lemma 1 and the Dominated Convergence Theorem we have

$$
\lim _{\varepsilon \rightarrow 0} J_{2}=\int_{0}^{1} \int_{\mathbf{R}^{2}} s^{-1 / 2} f\left(z_{1}\right) \phi\left(z_{1}\right) g\left(z_{2}\right) \phi\left(z_{2}\right) \lim _{\varepsilon \rightarrow 0} F_{\varepsilon}\left(z_{1}, z_{2}, s\right) d z_{1} d z_{2} d s .
$$

Setting $\varepsilon u=v$ in $F_{\varepsilon}\left(z_{1}, z_{2}, s\right)$ we get

$$
\begin{aligned}
F_{\varepsilon}\left(z_{1}, z_{2}, s\right)=\int_{2 \varepsilon}^{1-s}[ & \int_{\mathbf{R}^{2}} g\left(z_{3}\right) \frac{f^{\prime \prime}\left(\theta_{v}\right)}{2}\left(\beta_{2} \varepsilon^{-1 / 2} z_{2}+\beta_{3} \varepsilon^{-1 / 2} z_{3}\right)^{2} \\
& \left.\times \phi\left(z_{3}\right) \phi\left(z_{4}\right) d z_{3} d z_{4}\right] d v I_{[(M+2) \varepsilon, 1-2 \varepsilon]}(s)
\end{aligned}
$$

But $\theta_{v}$ tends to $z_{1}+\sqrt{v} z_{4}$ and both $\beta_{2} \varepsilon^{-1 / 2}$ and $\beta_{3} \varepsilon^{-1 / 2}$ converge to $2^{-1}\|\varphi\|_{2}^{-1}$ when $\varepsilon$ goes to zero, so using again the Dominated Convergence Theorem one has

$$
\begin{aligned}
\lim _{\varepsilon \rightarrow 0} J_{2}= & \int_{0}^{1} \int_{\mathbf{R}^{4}} \frac{f\left(z_{1}\right)}{8 \sqrt{s}} \phi\left(z_{1}\right) g\left(z_{2}\right) \phi\left(z_{2}\right) \\
& \times \int_{0}^{1-s} g\left(z_{3}\right) f^{\prime \prime}\left(z_{1}+\sqrt{v} z_{4}\right) \\
& \quad \times\|\varphi\|_{2}^{-2}\left(z_{2}+z_{3}\right)^{2} \phi\left(z_{3}\right) \phi\left(z_{4}\right) d v d z_{1} d z_{2} d z_{3} d z_{4} d s=0,
\end{aligned}
$$

since

$$
\int_{-\infty}^{+\infty} g(x) \phi(x) d x=\int_{-\infty}^{+\infty} x g(x) \phi(x) d x=0
$$

F o r $J_{3}$. A similar argument holds so that $J_{3}$ tends to zero when $\varepsilon$ goes to zero.

For cases 2 and 3 , using the fact that the measure of the integration domain is bounded by $C \varepsilon$ and a uniform bound in $\varepsilon$ for the integrand (which can be obtained by the previous calculations and Lemma 1), we get that both tend to zero when $\varepsilon$ goes to zero.

Adding the limits for the three cases we obtain the result. 
c. A s y mptotic convergence of $\left(X_{t}^{\epsilon}, S_{t}^{\varepsilon}\right)$.

c. $\alpha$. Preli minary result. Having in mind the theorem for the increments we use a more general function $g$. Let then $z$ be a continuous function verifying $|z(x)| \leqslant C\left(1+|x|^{n}\right)$ for some $n \in \mathbf{N}, \mathbf{E}[z(\xi)]=0$, $\mathbf{E}[\xi z(\xi)]=0$, where $\xi$ is a standard Gaussian random variable. Let

$$
M_{t}^{\varepsilon}(z)=\varepsilon^{-1 / 2} \int_{0}^{t} z\left(\dot{Y}_{s}^{\varepsilon}\right) I_{[M \varepsilon, 1]}(s) d s .
$$

Observe that $S_{t}^{\varepsilon}=M_{t}^{\varepsilon}(g)$. For $0 \leqslant t \leqslant 1$, we want to prove first that $M_{t}^{\varepsilon}(z)$ converges weakly to $\sigma(z) W_{t}$ (where $\sigma(z)$ will be defined below) when $\varepsilon$ goes to zero. To this end let's recall the following theorem proved in $[2, \mathrm{p} .25]$.

Theorem 3. Suppose that $\left\{Y_{n}\right\}$ has asymptotically independent increments, that $\left\{Y_{n}^{2}(t): n=1,2, \ldots\right\}$ is uniformly integrable for each $t$, and that $\mathbf{E}\left\{Y_{n}(t)\right\} \longrightarrow 0$ and $\mathbf{E}\left\{Y_{n}^{2}(t)\right\} \longrightarrow t$ as $n \rightarrow \infty$ for each $t$; suppose finally that the distributions of $Y_{n}$ are tight. Then $Y_{n}$ converges weakly to $W$ a standard Brownian motion.

We will apply this theorem to $M_{t}^{\varepsilon}(z)$, with the obvious modification that enables us to work with $\varepsilon \rightarrow 0$ instead of sequences.

Let $\left[t_{1}, t_{2}\right]$ and $\left[t_{3}, t_{4}\right]$ be two intervals in $[0,1]$ such that $t_{3}-t_{2}=d \geqslant$ $2 \varepsilon>0$ for $\varepsilon$ small enough, then $M_{t_{2}}^{\varepsilon}(z)-M_{t_{1}}^{\varepsilon}(z)$ and $M_{t_{4}}^{\varepsilon}(z)-M_{t_{3}}^{\varepsilon}(z)$ are independent.

By using that $z$ is centered we get $\mathbf{E}\left(M_{t}^{\varepsilon}(z)\right)=0$ and we claim that $\mathbf{E}\left(M_{t}^{\varepsilon}(z)\right)^{2} \longrightarrow \sigma^{2}(z) t$. In the case $z=g$ this was the result proved in b. taking $f \equiv 1$. For general $z$, using the same notation as in b., we have for $t>0$

$$
\mathbf{E}\left(M_{t}^{\varepsilon}(z)\right)^{2}=\varepsilon^{-1} \int_{M \varepsilon}^{t} \int_{M \varepsilon}^{t} \mathbf{E}\left[z\left(\dot{Y}_{s}^{\varepsilon}\right) z\left(\dot{Y}_{s^{\prime}}^{\varepsilon}\right)\right] d s^{\prime} d s .
$$

We consider the change of variable $s^{\prime}=s+\varepsilon u$ :

$$
\mathrm{E}\left(M_{t}^{\varepsilon}(z)\right)^{2}=\int_{M \varepsilon}^{t} \int_{(M \varepsilon-s) / \varepsilon}^{(t-s) / \varepsilon} \mathrm{E}\left[z\left(\dot{Y}_{s}^{\varepsilon}\right) z\left(\dot{Y}_{s+\varepsilon u}^{\varepsilon}\right)\right] d u d s
$$

We have again the three cases noted in $\mathrm{b}$.

(1) $(M+2) \varepsilon \leqslant s \leqslant t-2 \varepsilon$,

(2) $t-2 \varepsilon \leqslant s$,

(3) $s \leqslant(M+2) \varepsilon$.

The important case is the first one. Remember the decomposition made in b. into three integrals $J_{1}, J_{2}$ and $J_{3}$. We note that only $J_{1}$ is different from zero. The other two are zero because of the independence between $\dot{Y}_{s}^{\varepsilon}$ and $\dot{Y}_{s^{\prime}}^{\varepsilon}$ when $\left|s-s^{\prime}\right|>2 \varepsilon$. Using the Dominated Convergence Theorem and 
uniform integrability we get

$$
\begin{aligned}
J_{1} & =\int_{(M+2) \varepsilon}^{t-2 \varepsilon} \int_{-2}^{2} \mathbf{E}\left[z\left(\dot{Y}_{s}^{\varepsilon}\right) z\left(\dot{Y}_{s+\varepsilon u}^{\varepsilon}\right)\right] d u d s \\
& \longrightarrow t \int_{-2}^{2} \mathbf{E}\left[z\left(Z_{1}(u)\right) z\left(Z_{2}(u)\right)\right] d u=t \sigma^{2}(z),
\end{aligned}
$$

$\left(Z_{1}(u), Z_{2}(u)\right)$ is a two-dimensional Gaussian vector with zero mean and covariance matrix $\Gamma(u)$. Finally we prove tightness and uniform integrability.

$$
M_{t}^{\varepsilon}(z)-M_{s}^{\varepsilon}(z)=\varepsilon^{-1 / 2} \int_{s}^{t} z\left(\dot{Y}_{u}^{\varepsilon}\right) I_{[M \varepsilon, 1]}(u) d u=\varepsilon^{-1 / 2} \sum_{k=0}^{N(\varepsilon)-1} Z_{k}^{\varepsilon}+P_{t, s}^{\varepsilon}
$$

where

$$
N(\varepsilon)=\operatorname{Ent}\left\{2^{-1} \varepsilon^{-1}(t-s)\right\}, \quad Z_{k}^{\varepsilon}=\int_{s+2 k \varepsilon}^{s+2(k+1) \varepsilon} z\left(\dot{Y}_{u}^{\varepsilon}\right) I_{[M \varepsilon, 1]}(u) d u
$$

and

$$
P_{t, s}^{\varepsilon}=\varepsilon^{-1 / 2} \int_{s+2 N(\varepsilon) \varepsilon}^{t} z\left(\dot{Y}_{u}^{\varepsilon}\right) I_{[M \varepsilon, 1]}(u) d u .
$$

The random variables $Z_{k}^{\varepsilon}$ are 1 -dependent.

By using Lemma 1 , we obtain $\mathbf{E}\left[P_{t, s}^{\varepsilon}\right]^{4} \leqslant C|t-s|^{2}$. We shall prove that

$$
\mathbf{E}\left[\varepsilon^{-1 / 2} \sum_{k=0}^{N(\varepsilon)-1} Z_{k}^{\varepsilon}\right]^{4} \leqslant C|t-s|^{2}
$$

R e $\mathrm{m}$ a r k. $Z_{k}^{\varepsilon}$ is not necessarily stationary here because $s$ is arbitrary; but $Z_{k}^{\varepsilon}$ is centered because $\dot{Y}_{s}^{\varepsilon}$ is a standard Gaussian variable on $\{s>M \varepsilon\}$.

$$
\mathbf{E}\left[\varepsilon^{-1 / 2} \sum_{k=0}^{N(\varepsilon)-1} Z_{k}^{\varepsilon}\right]^{4}=\varepsilon^{-2} \sum_{k_{1}, k_{2}, k_{3}, k_{4}=0}^{N(\varepsilon)-1} \mathbf{E}\left[Z_{k_{1}}^{\varepsilon} Z_{k_{2}}^{\varepsilon} Z_{k_{3}}^{\varepsilon} Z_{k_{4}}^{\varepsilon}\right]
$$

We can suppose that $k_{1} \leqslant k_{2} \leqslant k_{3} \leqslant k_{4}$.

1. If $k_{4}-k_{3} \geqslant 2$ then $Z_{k_{4}}^{\varepsilon}$ is independent of $Z_{k_{1}}^{\varepsilon}, Z_{k_{2}}^{\varepsilon}$ and $Z_{k_{3}}^{\varepsilon}$ so

$$
\mathbf{E}\left[Z_{k_{1}}^{\varepsilon} Z_{k_{2}}^{\varepsilon} Z_{k_{3}}^{\varepsilon} Z_{k_{4}}^{\varepsilon}\right]=\mathbf{E}\left[Z_{k_{1}}^{\varepsilon} Z_{k_{2}}^{\varepsilon} Z_{k_{3}}^{\varepsilon}\right] \mathbf{E}\left[Z_{k_{4}}^{\varepsilon}\right]=0
$$

because $Z_{k_{4}}^{\varepsilon}$ is centered.

2. If $0 \leqslant k_{4}-k_{3} \leqslant 1$ (then $k_{4}$ is a function of $k_{3}$ ).

2.a. If $k_{3}-k_{2} \geqslant 2$, then $Z_{k_{3}}^{\varepsilon}$ and $Z_{k_{4}}^{\varepsilon}$ are independent of $Z_{k_{1}}^{\varepsilon}$ and $Z_{k_{2}}^{\varepsilon}$ so

$$
\mathbf{E}\left[Z_{k_{1}}^{\varepsilon} Z_{k_{2}}^{\varepsilon} Z_{k_{3}}^{\varepsilon} Z_{k_{4}}^{\varepsilon}\right]=\mathbf{E}\left[Z_{k_{1}}^{\varepsilon} Z_{k_{2}}^{\varepsilon}\right] \mathbf{E}\left[Z_{k_{3}}^{\varepsilon} Z_{k_{4}}^{\varepsilon}\right]
$$

2.a. $\alpha$. If $k_{2}-k_{1} \geqslant 2$ then $Z_{k_{2}}^{\varepsilon}$ is independent of $Z_{k_{1}}^{\varepsilon}$ and $(1)=0$. 
2.a. $\beta$. If $0 \leqslant k_{2}-k_{1} \leqslant 1$ (then $k_{2}$ is a function of $k_{1}$ ) then the sum takes place only over two indices. Using Schwarz's inequality and the fact that $\mathbf{E}\left[Z_{k}^{\varepsilon}\right]^{2 p} \leqslant \varepsilon^{2 p}$ for all $p \geqslant 0$ one has that the sum is less than

$$
C \varepsilon^{-2} N^{2}(\varepsilon) \varepsilon^{4} \leqslant C|t-s|^{2}
$$

2.b. If $0 \leqslant k_{3}-k_{2} \leqslant 1$ (then $k_{3}$ is a function of $k_{2}$ ). The sum takes place only over two indices, as in 2.a. $\beta$ it is less than $C|t-s|^{2}$ and the result holds.

The uniform integrability is a consequence of $\mathrm{E}\left(M_{t}^{\varepsilon}(z)\right)^{4} \leqslant C t^{2} \leqslant C$.

c. $\beta$. As y mptotic independence between $X(t)$ a n d $W(t)$.

c. $\beta .1$. T i g h t $\mathrm{n}$ e s s. We know that $X_{\bullet}^{\varepsilon} \rightarrow X_{\bullet}$ a.e. and $M_{\bullet}^{\varepsilon}(z) \longrightarrow$ $\sigma(z) W_{\bullet}$ weakly. This implies that the bidimensional sequence $\left(X_{\bullet}^{\varepsilon}, M_{\bullet}^{\varepsilon}(z)\right)$ is tight in $C[0 ; 1] \times C[0 ; 1]$.

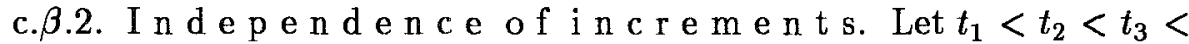
$t_{4}$ and consider the two random vectors $\left(X_{t_{2}}^{\varepsilon}-X_{t_{1}}^{\varepsilon}, M_{t_{2}}^{\varepsilon}(z)-M_{t_{1}}^{\varepsilon}(z)\right)$ and $\left(X_{t_{4}}^{\varepsilon}-X_{t_{3}}^{\varepsilon}, M_{t_{4}}^{\varepsilon}(z)-M_{t_{3}}^{\varepsilon}(z)\right)$. We can suppose without loss of generality that $t_{3}-t_{2}>3 \varepsilon$ and $t_{1}>M \varepsilon$. To study the independence between these two vectors, observe that the first is in

$$
\mathcal{F}_{t_{2}+\varepsilon}=\sigma\left\{X_{s}: s \leqslant t_{2}+\varepsilon\right\} \text {. }
$$

Furthermore, if $X_{s}$ belongs to this $\sigma$-algebra it holds that

$$
\mathbf{E}\left(X_{s}\left[c_{1}\left(X_{t_{4}}^{\varepsilon}-X_{t_{3}}^{\varepsilon}\right)+c_{2} \dot{X}_{t}^{\varepsilon}\right]\right)=0
$$

where $t_{3}-\varepsilon \leqslant t$. This fact implies the independence between $\mathcal{F}_{t_{2}+\varepsilon}$ and the $\sigma$-algebra generated by the Gaussian vectors

$$
\left(X_{t_{4}}^{\varepsilon}-X_{t_{3}}^{\varepsilon}, \dot{X}_{t}^{\varepsilon}, t_{3}-\varepsilon \leqslant t\right)
$$

Given that $\left(X_{t_{4}}^{\varepsilon}-X_{t_{3}}^{\varepsilon}, M_{t_{4}}^{\varepsilon}(z)-M_{t_{3}}^{\varepsilon}(z)\right)$ belongs to this $\sigma$-algebra, the mutual independence holds.

c. $\beta .3$. L i m it identification. Let $Y$ be any limit point of $\left(X_{\bullet}^{\varepsilon}, M_{\bullet}^{\varepsilon}(z)\right)$. From the results of c. $\beta .2, Y$ will be a vector process having independent increments and finite second moment, thus it must be Gaussian. We have, therefore, only to identify its covariance. We have calculated the variances before. The first coordinate is a Brownian motion and the second one has $\sigma^{2}(z) t$ as variance. It remains only to compute

$$
\mathbf{E}\left\{Y_{1} Y_{2}\right\}=\lim _{\varepsilon \rightarrow 0} \mathbf{E}\left\{X_{t}^{\varepsilon} M_{t}^{\varepsilon}(z)\right\}=0 \text {. }
$$

This last result is a consequence of $\mathbf{E}[\xi z(\xi)]=0$. We have shown that all the limit points of $\left(X_{\bullet}^{\varepsilon}, M_{\bullet}^{\varepsilon}(z)\right)$ converge weakly to the same limit $\left(X_{\bullet}, \sigma(z) W_{\bullet}\right)$. Therefore the sequence converges weakly and the two coordinates of the limit 
processes are independent. By taking $z=g$ this implies the same result for $\left(X_{\bullet}^{\varepsilon}, S_{\bullet}^{\varepsilon}\right)$.

d. A s y m ptotic convergence of $T_{1}$. In this section we will work with $W$, the Wiener processes limit of $S_{t}^{\varepsilon}$. Recall that

$$
Z^{n}(f)=\sum_{i=1}^{n} f\left(X_{(i-1) / n}\right) \sigma\left[W_{i / n}-W_{(i-1) / n}\right]
$$

We can prove using standard arguments that there exists $Y \in L^{2}(\Omega)$ such that $\left\|Z^{n}(f)-Y\right\|_{2} \longrightarrow 0$ as $n$ goes to infinity.

To show the convergence of $T_{1}$, it is enough to prove that

$$
\lim _{n \rightarrow \infty} \lim _{\varepsilon \rightarrow 0}\left\|T_{1}-Z_{\varepsilon}^{n}(f)\right\|_{2}=0
$$

We have

$$
\mathbf{E}\left[T_{1}-Z_{\varepsilon}^{n}(f)\right]^{2}=\mathbf{E}\left[T_{1}\right]^{2}+\mathbf{E}\left[Z_{\varepsilon}^{n}(f)\right]^{2}-2 \mathbf{E}\left[T_{1} Z_{\varepsilon}^{n}(f)\right]
$$

We have seen in b. that $\mathbf{E}\left[T_{1}\right]^{2} \longrightarrow \sigma^{2} \sigma_{0}^{2}$ as $\varepsilon$ goes to zero and a similar proof holds for $\mathbf{E}\left[T_{1} Z_{\varepsilon}^{n}(f)\right]$, i.e.,

$$
\mathbf{E}\left[T_{1} Z_{\varepsilon}^{n}(f)\right] \longrightarrow \sigma^{2} \sum_{i=1}^{n} \int_{(i-1) / n}^{i / n} \mathbf{E}\left[f\left(X_{s}\right) f\left(X_{(i-1) / n}\right)\right] d s
$$

when $\varepsilon$ goes to zero. The last term tends to $\sigma^{2} \sigma_{0}^{2}$ when $n$ goes to infinity thanks to Lemma 1 and the Dominated Convergence Theorem.

To end the proof, we show that

$$
\begin{gathered}
\lim _{n \rightarrow \infty} \lim _{\varepsilon \rightarrow 0} \mathbf{E}\left[Z_{\varepsilon}^{n}(f)\right]^{2}=\sigma^{2} \sigma_{0}^{2} \\
\mathbf{E}\left[Z_{\varepsilon}^{n}(f)\right]^{2}=\sum_{i=1}^{n} \sum_{j=1}^{n} \mathbf{E}\left[f\left(X_{(i-1) / n}^{\varepsilon}\right) f\left(X_{(j-1) / n}^{\varepsilon}\right)\left(S_{i / n}^{\varepsilon}-S_{(i-1) / n}^{\varepsilon}\right)\right. \\
\longrightarrow \sigma^{2} \sum_{i=1}^{n} \sum_{j=1}^{n} \mathbf{E}\left[f\left(X_{(i-1) / n}^{\varepsilon}\right) f\left(X_{(j-1) / n}\right)\right] \\
\times \mathbf{E}\left\{\left[W_{i / n}-W_{(i-1) / n}^{\varepsilon}\right]\left[W_{j / n}-W_{(j-1) / n}\right]\right\} \\
=\frac{\sigma^{2}}{n} \sum_{i=1}^{n} \mathbf{E}\left[f\left(X_{(i-1) / n}\right)\right]^{2}
\end{gathered}
$$


as $\varepsilon$ goes to zero. To see this, on the one hand we have as in [1], and thanks to $c . \beta$, that

$$
\begin{aligned}
& \left(X_{(i-1) / n}^{\varepsilon}, X_{(j-1) / n}^{\varepsilon}, S_{i / n}^{\varepsilon}-S_{(i-1) / n}^{\varepsilon}, S_{j / n}^{\varepsilon}-S_{(j-1) / n}^{\varepsilon}\right) \\
& \quad \longrightarrow\left(X_{(i-1) / n}, X_{(j-1) / n}, W_{i / n}-W_{(i-1) / n}, W_{j / n}-W_{(j-1) / n}\right)
\end{aligned}
$$

weakly as $\varepsilon$ goes to zero and on the other hand, by Hölder's inequality and Lemma 1 , for $\delta<1$ :

$$
\begin{aligned}
& \mathbf{E}\left|f\left(X_{(i-1) / n}^{\varepsilon}\right) f\left(X_{(j-1) / n}^{\varepsilon}\right)\left(S_{i / n}^{\varepsilon}-S_{(i-1) / n}^{\varepsilon}\right)\left(S_{j / n}^{\varepsilon}-S_{(j-1) / n}^{\varepsilon}\right)\right|^{1+\delta} \\
& \quad \leqslant C\left\{\mathbf{E}\left[S_{i / n}^{\varepsilon}-S_{(i-1) / n}^{\varepsilon}\right]^{4}\right\}^{(1+\delta) / 4}\left\{\mathbf{E}\left[S_{j / n}^{\varepsilon}-S_{(j-1) / n}^{\varepsilon}\right]^{4}\right\}^{(1+\delta) / 4} \leqslant C .
\end{aligned}
$$

The uniform integrability theorem yields the result.

Finally, we remark that

$$
n^{-1} \sum_{i=1}^{n} \mathbf{E}\left[f\left(X_{(i-1) / n}\right)\right]^{2} \rightarrow \sigma_{0}^{2}
$$

when $n$ goes to infinity. This yields Theorem 1 .

e. P r o of of The ore m 2. Let's begin by proving (i). We have only to observe the following: the function $z$ is now $g_{\beta}(x)=|x|^{\beta}-\mathbf{E}|\xi|^{\beta}$, where $\xi$ is a standard Gaussian r.v., and $\Delta_{\varepsilon}(t)=\varepsilon^{1 / 2} \dot{X}_{\varepsilon}(t)$ by taking $\varphi=$ $I_{[-1,0]}$ (cf. [10]) and $f \equiv 1$. The asymptotic variance is

$$
\sigma_{\beta}^{2}=\int_{-\infty}^{+\infty} \mathrm{E}\left[g_{\beta}\left(Z_{1}(u)\right) g_{\beta}\left(Z_{2}(u)\right)\right] d u .
$$

To prove (ii) let's define

$$
z_{x}(y)=I_{(-\infty, x]}(y)-\Phi(x)+\phi(x) y
$$

This function verifies the condition given in c. $\alpha$. except for the continuity $\left(\varphi=I_{[-1,0]}\right.$ and $\left.f \equiv 1\right)$. However, this property is only important to compute the limit variance but it is straightforward to do the computations in this case. Hence we have

$$
M_{t}^{\varepsilon}\left(z_{x}\right)=\varepsilon^{-1 / 2} \int_{0}^{t} z_{x}\left(\dot{Y}_{s}^{\varepsilon}\right) I_{[M \varepsilon, 1]}(s) d s \longrightarrow \sigma\left(z_{x}\right) W_{t}
$$

weakly in $C[0,1]$. We get also that the two processes $X_{\bullet}$ and $W_{\bullet}$ are inde- 
pendent. Thus

$$
\begin{aligned}
B_{\varepsilon}(t, x)= & M_{t}^{\varepsilon}\left(z_{x}\right)-\phi(x) \int_{M \varepsilon}^{t} \dot{X}_{s}^{\varepsilon} d s \\
& +\lambda\left\{s \leqslant M \varepsilon, \Delta_{\varepsilon}(s) \leqslant x\right\}-M \Phi(x) \sqrt{\varepsilon} \\
= & M_{t}^{\varepsilon}\left(z_{x}\right)-\phi(x)\left(X_{t}^{\varepsilon}-X_{M \varepsilon}^{\varepsilon}\right) \\
& +\lambda\left\{s \leqslant M \varepsilon, \Delta_{\varepsilon}(s) \leqslant x\right\}-M \Phi(x) \sqrt{\varepsilon}
\end{aligned}
$$

By using the joint weak convergence of $\left(X^{\varepsilon}(\bullet), M_{\bullet}^{\varepsilon}\left(z_{x}\right)\right)$ to $\left(X_{\bullet}, \sigma\left(z_{x}\right) W_{\bullet}\right)$ we get that

$$
B_{\varepsilon}(t, x) \longrightarrow \sigma\left(z_{x}\right) W_{t}-\phi(x) X_{t}=\eta(t, x)
$$

The Hermite expansion of the function $I_{(-\infty, x]}(y)$ and Mehler's formula provide a more explicit expression for the limit variance:

$$
\begin{aligned}
\mathbf{E}\left\{B_{\varepsilon}^{2}(t, x)\right\} & \longrightarrow 2 t\left[\frac{\phi^{2}(x)}{2}+\phi^{2}(x) \sum_{n=2}^{\infty} \frac{H_{n-1}^{2}(x)}{(n+1) !}\right] \\
& =2 t \phi^{2}(x) \sum_{n=1}^{\infty} \frac{H_{n-1}^{2}(x)}{(n+1) !}
\end{aligned}
$$

\section{REFERENCES}

1. Berzin C., León J. R., Ortega J. Level crossings and local time for regularized Gaussian processes. Prépublication Université de Paris-Sud, 1993.

2. Billingsley P. Weak Convergence of Measures. Regional Conference Series in Applied Mathematics. Philadelphia: SIAM, 1987.

3. Csaki E., Csörgő M., Földes A., Révész P. Brownian local time approximated by a Wiener sheet. - Ann. Probab. 1989, v. 17, № 2, p. 516-537.

4. Borodin A. N. On the character of convergence to Brownian local time I. $-\mathrm{Z}$. Wahrscheinlichkeitstheor. verw. Geb., 1986, B. 72, S. 231-250.

5. Borodin $A$. N. On the character of convergence to Brownian local time II. - Z Wahrscheinlichkeitstheor. verw. Geb., 1986, B. 72, S. 251-277.

6. Бородин $A$. H. Броуновское локальное время. - Успехи матем. наук, 1989, т. 44, B. 2 , c. $7-48$.

7. Kasahara $Y$. On Lévy downcrossing theorem. - Proc. Japan Acad. Ser. A Math. Sci., 1980 , v. 56, № 10, p. 455-458.

8. Revuz D., Yor M. Continuous Martingales and Brownian motion. - Berlin: SpringerVerlag, 1991, $560 \mathrm{p}$.

9. Wschebor $M$. Surfaces aléatoires: mesure géométrique des ensembles de niveau. (Lect. Notes Math., V. 1147.) Berlin: Springer-Verlag, 1985.

10. Wschebor M. Sur les accroissements du processus de Wiener. - C. R. Acad. Sci. Paris. 1992, v. 315, p. 1293-1296. 